\title{
Phase II Study of Weekly Amrubicin for Refractory or Relapsed Non-small Cell Lung Cancer
}

\author{
CHIYOE KITAGAWA ${ }^{1,2^{*}}$, MASAHIRO IWASAKU ${ }^{3 *}$, YOSHIHITO KOGURE $^{1}$, \\ MASAHIKO ANDO $^{4}$, HIROSHIGE YOSHIOK ${ }^{3,5}$, AKIHIRO NISHIYAMA ${ }^{3}$, NAOKI WATANABE ${ }^{3}$, \\ KEI KUNIMASA ${ }^{3}$, KAZUYA TSUBOUCHI ${ }^{3}$, SAORI OKA ${ }^{2}$ and HIDEO SAKA ${ }^{1,2}$ \\ ${ }^{1}$ Department of Respiratory Medicine, National Hospital Organization Nagoya Medical Center, Nagoya, Japan; \\ ${ }^{2}$ Department of Medical Oncology, National Hospital Organization Nagoya Medical Center, Nagoya, Japan; \\ ${ }^{3}$ Department of Respiratory Medicine, Kurashiki Central Hospital, Kurashiki, Japan; \\ ${ }^{4}$ Center for Advanced Medicine and Clinical Research, Nagoya University Hospital, Nagoya, Japan; \\ ${ }^{5}$ Kansai Medical University Hospital, Department of Respiratory Oncology, Osaka, Japan
}

\begin{abstract}
Background: Amrubicin is usually administered on days 1-3 every 3 weeks by intravenous infusion. However, it causes severe hematological toxicity, especially febrile neutropenia. It was reported that weekly administration confers higher dose intensity, less severe adverse events, and anti-tumor activity that is as effective as that of treatment with a conventional schedule. Patients and Methods: Weekly amrubicin was administered at a dose of $60 \mathrm{mg} / \mathrm{m}^{2}$ on days 1 and 8 every 3 weeks. The primary endpoint was overall response rate. Results: A total of 33 patients were enrolled. The overall response rate was $6.1 \%$ (95\% confidence interval $(C I)=0.7-20.2 \%)$ and the disease control rate after 2 months was $51.5 \%$. The median progression-free survival was 2.93 months. Febrile neutropenia was observed in only two patients. Conclusion: The primary endpoint was not met in this study. However, weekly amrubicin achieved a high disease control rate and good tolerability.
\end{abstract}

Amrubicin is a potent topoisomerase II inhibitor and promising agent for both small-cell lung cancer (SCLC) and non-small cell lung cancer (NSCLC). Therapeutic strategy has been mainly developed for SCLC and amrubicin using

This article is freely accessible online.

*These Authors contributed equally to this study.

Correspondence to: Chiyoe Kitagawa, MD, Ph.D., Department of Respiratory Medicine, National Hospital Organization Nagoya Medical Center, Nagoya, Japan. Tel: +81 529511111, Fax: +81 529510664,e-mail: kitagawc@nnh.hosp.go.jp

Key Words: Amrubicin, non-small cell lung cancer, refractory, relapsed, phase II.
$40 \mathrm{mg} / \mathrm{m}^{2}$ on days $1-3$ every 3 weeks (standard amrubicin) is widely used for SCLC patients for second-line setting or later.

In NSCLC, the late-phase II trial reported a response rate of $26.7 \%$ for patients treated with prior chemotherapy $(1,2)$. However, amrubicin did not demonstrate its greater efficacy compared to docetaxel for patients with NSCLC treated in the second-line setting (3). Furthermore, standard amrubicin causes severe, occasionally fatal, hematological toxicity, especially febrile neutropenia $(\mathrm{FN})$.

For administration method, amrubicin was initially investigated at a dose of once a day every 3 weeks (recommended dose was $100 \mathrm{mg} / \mathrm{m}^{2}$ ) in the phase I study (4), then divided into then doses on day 1-3 (1). However, in actual clinical application of standard amrubicin, the treatment schedule tends to be delayed due to myelosuppression from the previous course of amrubicin treatment, and the total dose tends to be lower than expected due to the discontinuation of amrubicin because of myelosuppression. It is suggested that toxicity may be kept low and total dosage may be increased by performing weekly administration and weekly administration of other chemotherapeutic reagents, such as paclitaxel, has been reported in a clinical trial (5).

Thus, we conducted a phase I study of weekly amrubicin and the recommended dose was $60 \mathrm{mg} / \mathrm{m}^{2}$ weekly on days 1 and 8 every 3 weeks (6). Here we conducted a phase II trial to evaluate the efficacy and toxicity of weekly amrubicin for advanced NSCLC.

\section{Patients and Methods}

Study design. This was an open-label, multicenter, phase II study. Primary inclusion criteria were histologically/cytologically-proven NSCLC, tumor progression or refractory disease after performing one or two chemotherapy regimens (excluding gefitinib or 
Table I. Patient demographics.

\begin{tabular}{lc}
\hline & Value \\
\hline No. of patients & \\
Total & 33 \\
Gender, n (\%) & \\
Male & $21(63.6)$ \\
Female & $12(36.4)$ \\
Age (years) & \\
Median (range) & $67(38-80)$ \\
ECOG PS, n (\%) & \\
0 & $8(24.2)$ \\
1 & $22(66.7)$ \\
2 & $3(9.1)$ \\
Histology, $\mathrm{n}(\%)$ & \\
Adenocarcinoma & $24(72.7)$ \\
Squamous & $7(21.2)$ \\
Non-small cell & $2(6.1)$ \\
No. of prior chemotherapies, $\mathrm{n}(\%)$ & \\
1 & $8(24.2)$ \\
2 & $21(63.6)$ \\
$3 *$ & $4(12.1)$ \\
Prior therapies, n (\%) & \\
Surgery & $1(3.0)$ \\
Radiotherapy & $1(3.0)$ \\
EGFR mutation status, n $(\%)$ & \\
Negative & $23(69.7)$ \\
Ex19 del & $2(6.1)$ \\
L858R & $2(6.1)$ \\
L861Q & $1(3.0)$ \\
L858R T790M & $1(3.0)$ \\
Unknown & $4(12.1)$ \\
\hline & \\
\hline & \\
\hline &
\end{tabular}

*One EGFR-TKI regimen was included. ECOG PS, Eastern Cooperative Oncology Group performance status; EGFR, epidermal growth factor receptor.

erlotinib), age $\geq 20$ years, Eastern Cooperative Oncology Group (ECOG) performance status of 0 to 2 , and adequate main organ functions. Primary exclusion criteria were contraindication for amrubicin, carcinomatous pleuritis, pericarditis, peritonitis and other metastasis with local therapy indication, in a course of receiving radiotherapy, serious medical complication, and infection.

This study was conducted in accordance with the Declaration of Helsinki and Guideline for Good Clinical Practice. The study protocol was reviewed and approved by the individual institutional review board, and written informed consent was obtained from all patients. This study has been registered in the UMIN Clinical Trials Registry (UMIN-CTR, URL: http://www.umin.ac.jp/ctr/), number UMIN000002570.

Amrubicin was administered at the dose of $60 \mathrm{mg} / \mathrm{m}^{2}$ weekly (on days 1 and 8 every 3 weeks) until disease progression or intolerable toxicity was confirmed. Adverse events (AEs) were monitored and graded according to the National Cancer Institute Common Terminology Criteria for Adverse Events (CTCAE) version 3.0 (7).

The primary endpoint was the tumor response rate. The response rate was assessed according to Response Evaluation Criteria in Solid Tumors (RECIST), version 1.1 (8). Secondary endpoints were
Table II. Tumor response.

\begin{tabular}{lc}
\hline Response & $\mathrm{N}(\%)$ \\
\hline $\mathrm{CR}$ & 0 \\
$\mathrm{PR}$ & $2(6.1)$ \\
$\mathrm{SD}$ & $15(45.5)$ \\
$\mathrm{PD}$ & $12(36.4)$ \\
$\mathrm{NE}$ & $4(12.1)$ \\
\hline
\end{tabular}

$\mathrm{CR}$, Complete response; PR, partial response; $\mathrm{SD}$, stable disease; $\mathrm{PD}$, progression; NE, not evaluable.

Table III. Adverse events experienced by patients on weekly amrubicin.

\begin{tabular}{llc}
\hline Adverse event & \multicolumn{2}{c}{$\mathrm{n}(\%)$} \\
\hline Preferred term & Grade 3 & Grade 4 \\
Neutrophil count reduced & $5(15.2)$ & $12(36.4)$ \\
White blood cell count reduced & $7(21.2)$ & $5(15.2)$ \\
Anemia & $2(6.1)$ & $3(9.1)$ \\
Platelet count reduced & $2(6.1)$ & 0 \\
Febrile neutropenia & $2(6.1)$ & 0 \\
Anorexia & $5(15.2)$ & 0 \\
Malaise & $3(9.1)$ & 0 \\
Nausea & $2(6.1)$ & 0 \\
Infection (lung) & $2(6.1)$ & 0 \\
Hypoxia & $2(6.1)$ & $1(3.0)$ \\
Dyspnea & 0 & $1(3.0)$ \\
Pneumonitis & $1(3.0)$ & 0 \\
Fatigue & $1(3.0)$ & 0 \\
Hypoalbuminemia & $1(3.0)$ & 0 \\
\hline
\end{tabular}

No treatment-related deaths were reported.

adverse events, disease control rate (DCR) after 2 months of initial administration of amrubicin, progression-free survival (PFS) and overall survival (OS).

Statistical methods. Based on the results of second-/third-line regimens for advanced NSCLC $(9,10)$, the threshold response rate and the expected response rate were set as $10 \%$ and $30 \%$, respectively in expectation of an effect higher than with those regimens. Calculating the required number of cases based on Simon's two-stage design under the condition of significance level of 5\% (one-sided) and detection power of $85 \%, 14$ cases were required in the first stage. Moreover, an additional 13 cases would be required if one or more cases were judged as partial response (PR) or more by the attending doctor. In consideration of inappropriate cases and drop-out cases, the target number of cases was 32 for the whole study.

Analysis was performed of cases in which amrubicin was administered one or more times in this study, and the response rate and its exact $95 \%$ confidence interval (CI) were estimated. For timeto-event data, the Kaplan-Meier method was used to illustrate the survival curve and estimate the median survival time. For adverse events of grade 3 or more, the incidence rate was calculated. 

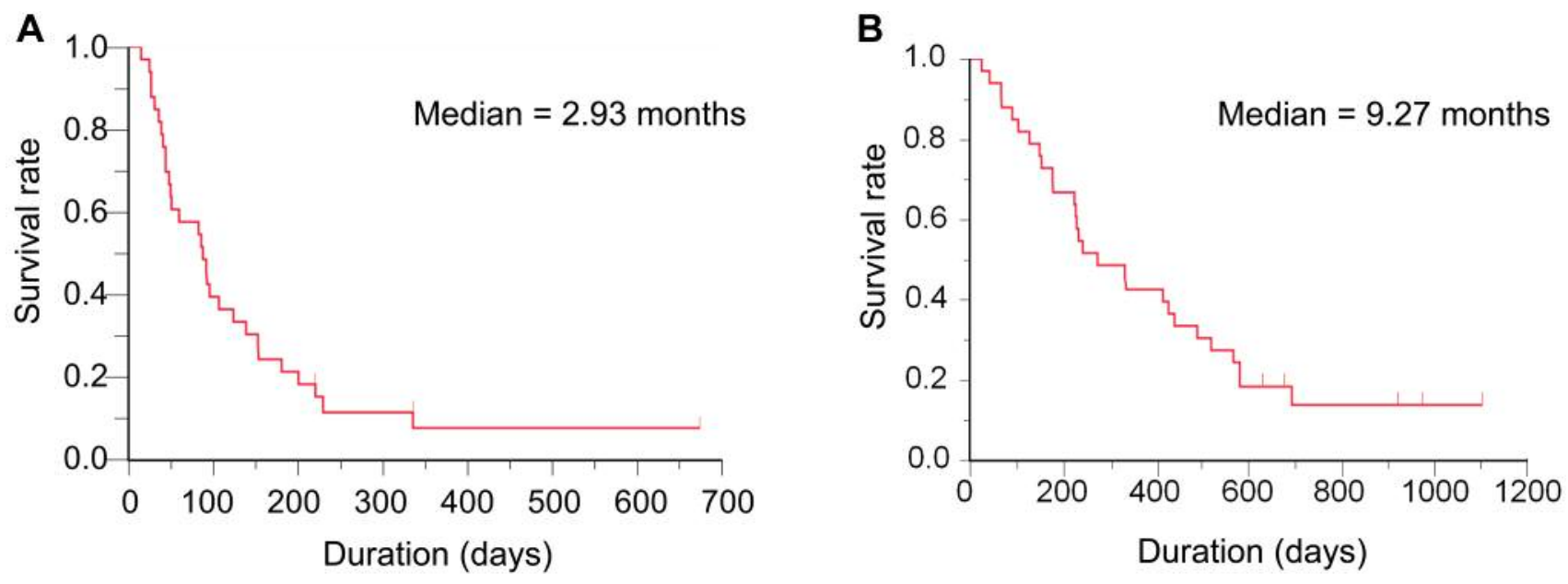

Figure 1. Survival of patients on weekly amrubicin. A: Progression-free survival; B: overall survival.

\section{Results}

A total of 33 patients were enrolled during the period from August 16, 2010 through May 11, 2012. The patients participated from two centers in Japan. Data cutoff was conducted on September 14, 2013. The median follow-up time was 9.27 months (0.93-36.8 months). Patient characteristics are shown in Table I. The median age was 67 years, two-thirds of the patients were male, $72 \%$ of them had adenocarcinoma, and $75.8 \%$ of the patients had received two or more prior chemotherapies. A median of three cycles (range $=1->23$ ) were administered.

The ORR was $6.1 \%(95 \% \mathrm{CI}=0.7-20.2 \%)$, which was lower than threshold response rate of $10 \%$ (Table II). The DCR after 2 months was $51.5 \%$. Thus, the primary endpoint was not met in this study. In terms of secondary endpoints, grade 3 or 4 hematological AEs were neutropenia (51.6\%), leukopenia $(36.4 \%)$, anemia $(15.2 \%)$ and thrombocytopenia $(6.1 \%)$. FN was seen in two patients $(6.1 \%)$. Regarding major grade 3 or 4 non-hematological AEs, anorexia $(15.2 \%)$, malaise $(9.1 \%)$, hypoxia $(9.1 \%)$ and nausea $(6.1 \%)$ were observed. Grade 3 pneumonitis occurred in one patient (3.0\%) and no treatment-related death was reported (Table III). The median PFS was 2.93 months and median OS was 9.27 months (Figure 1).

Subsequent treatment of patients on weekly amrubicin is shown in Table IV.

\section{Discussion}

In this study, the ORR of weekly amrubicin was $6.1 \%(95 \%$ $\mathrm{CI}=0.7-20.2 \%)$ and the primary endpoint was not met in patients with refractory or relapsed NSCLC. Docetaxel is the
Table IV. Subsequent treatment of patients on weekly amrubicin.

\begin{tabular}{ll}
\hline Treatment & $\mathrm{n}(\%)$ \\
\hline Chemotherapy (mono) & \\
$\quad$ Erlotinib & $9(28.1)$ \\
Docetaxel & $3(9.4)$ \\
Pemetrexed & $3(9.4)$ \\
Other & $4(12.5)$ \\
Platinum-containing regimen & $4(12.5)$ \\
Radiotherapy & $4(12.5)$ \\
Chemoradiotherapy & $1(3.1)$ \\
Molecular target drug & $1(3.1)$ \\
Strontium 89 & $1(3.1)$ \\
None & $2(6.3)$ \\
\hline
\end{tabular}

standard second-line cytotoxic agent for NSCLC, with ORR of $8.8 \%$, stable disease rate of $46.4 \%$, DCR of $55.0 \%$ and PFS of 2.9 months (9). Given that $75.8 \%$ of patients in this trial had received two or three prior chemotherapies, with a PFS of 2.93 months and DCR of $51.5 \%$, weekly amrubicin may be an alternative candidate for use as second-line salvage therapy. As for as safety is concerned, the frequency of FN was acceptable $(6.1 \%)$, considering that it was $12.7 \%$ in the trial using docetaxel (9).

There are several limitations in this trial. Firstly, it might be inadequate to choose the ORR as the primary endpoint, since three-quarters of patients had received two or three prior chemotherapies. PFS would have been better as the primary endpoint. Secondly, immune checkpoint inhibitors are administered in first-/second-line settings in current therapeutic strategies. However, no patients received immune 
checkpoint inhibitors in this trial and therefore we were unable to analyze the efficacy of weekly amrubicin after therapy with immune checkpoint inhibitors.

In conclusion, the primary endpoint was negative in this trial. However, this regimen showed comparable PFS and lower rates of FN. Weekly amrubicin can be an alternative regimen for salvage settings after second-line therapy.

\section{Acknowledgements}

The Authors thank all of the patients, their families, institutions and investigators participating in this study.

\section{References}

1 Sugiura T, Ariyoshi Y, Negoro S, Nakamura S, Ikegami H, Takada M, Yana $\mathrm{T}$ and Fukuoka M: Phase I/II study of amrubicin, a novel 9-aminoanthracycline, in patients with advanced non-small-cell lung cancer. Invest New Drugs 23: 331337, 2005.

2 Hanada M, Noguchi T and Murayama T: Profile of the antitumor effects of amrubicin, a completely synthetic anthracycline. Nihon Yakurigaku Zasshi 122: 141-150, 2003.

3 Yoshioka H, Katakami N, Okamoto H, Iwamoto Y, Seto T, Takahashi T, Sunaga N7, Kudoh S, Chikamori K, Harada M, Tanaka H, Saito H, Saka H, Takeda K, Nogami N, Masuda N, Harada T, Kitagawa H, Horio H, Yamanaka T, Fukuoka M, Yamamoto $\mathrm{N}$ and Nakagawa K: A randomized, open-label, phase III trial comparing amrubicin versus docetaxel in patients with previously treated non-small-cell lung cancer. Ann Oncol 28: 285-291, 2017.

4 Inoue K, Ogawa M, Horikoshi N, Mukaiyama T, Itoh Y, Imajoh K, Ozeki H, Nagamine D and Shinagawa K: Phase I and pharmacokinetic study of SM-5887, a new anthracycline derivative. Invest New Drugs 7: 213-218, 1989.

5 Belani CP, Ramalingam S, Perry MC, LaRocca RV, Rinaldi D, Gable PS and Tester WJ: Randomized, phase III study of weekly paclitaxel in combination with carboplatin versus standard every-3-weeks administration of carboplatin and paclitaxel for patients with previously untreated advanced non-small-cell lung cancer. J Clin Oncol 26: 468-473, 2008.
6 Kitagawa C, Saka H, Kajikawa S, Mori K, Oki M, Suzuki R: Phase I and pharmacologic study of weekly amrubicin in patients with refractory or relapsed lung cancer: Central Japan Lung Study Group (CJLSG) 0601 trial. Cancer Chemother Pharmacol 69: 1379-1385, 2012.

7 National Cancer Institute: Common Terminology Criteria for Adverse Events (CTCAE), Version 3.0. Available at: http://evs.nci.nih.gov/ftp1/CTCAE/About.html. Accessed on 10 October 2018.

8 Eisenhauer EA, Therasse P, Bogaerts J, Schwartz LH, Sargent D, Ford R, Dancey J, Arbuck S, Gwyther S, Mooney M, Rubinstein L, Shankar L, Dodd L, Kaplan R, Lacombe D and Verweij J: New response evaluation criteria in solid tumours: revised RECIST guideline (version 1.1). Eur J Cancer 45: 228247, 2009.

9 Hanna N, Shepherd FA, Fossella FV, Pereira JR, De Marinis F, von Pawel J, Gatzemeier U, Tsao TC, Pless M, Muller T, Lim HL, Desch C, Szondy K, Gervais R, Shaharyar, Manegold C, Paul S, Paoletti P, Einhorn L and Bunn PA Jr.: Randomized phase III trial of pemetrexed versus docetaxel in patients with non-small-cell lung cancer previously treated with chemotherapy. J Clin Oncol 22: 1589-1597, 2004.

10 Nokihara H, Lu S, Mok TSK, Nakagawa K, Yamamoto N, Shi YK, Zhang L, Soo RA, Yang JC, Sugawara S, Nishio M, Takahashi T, Goto K, Chang J, Maemondo M, Ichinose Y, Cheng Y, Lim WT, Morita S and Tamura T: Randomized controlled trial of S-1 versus docetaxel in patients with non-small-cell lung cancer previously treated with platinum-based chemotherapy (East Asia S-1 Trial in Lung Cancer). Ann Oncol 28: 2698-2706, 2017.

Received September 14, 2018 Revised October 17, 2018 Accepted October 23, 2018 